\title{
Importancia de los conceptos en el aprendizaje de la mecánica
}

\author{
HERNANDO GONZÁLEZ S.", CLOTARIO I. PERALTA G.**, YASMIDT VERA*
}

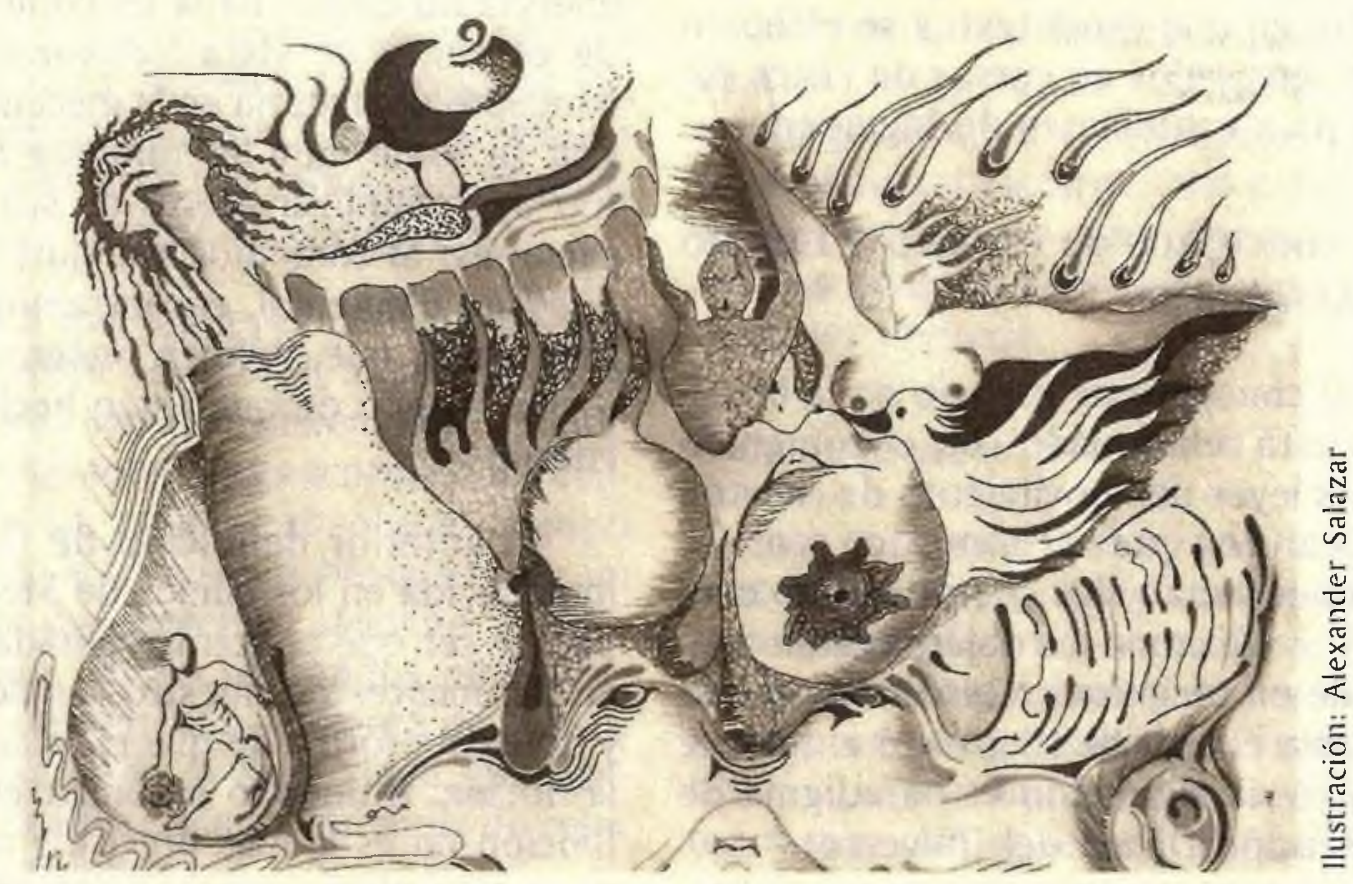

Introducción

as dificultades que tienen los estudiantes en el aprendizaje de la LFísica, se deben a diferentes factores. Queremos hacer hincapié en el papel preponderante que tiene la comprensión adecuada de los conceptos en el entendimiento del mundo físico. La Física se fundamenta en modelos, leyes, teorías, principios, postulados, los cuales tienen anexo un esquema conceptual que, si es bien entendido y aplicado mejorará sustancialmente el aprendizaje de esta ciencia.

\footnotetext{
- Profesor Universidad Surcolombiana

$\infty$ Profesor Universidad Surcolombiana

Estudiante de Matemáticas y Física, Universidad Surcolombiana
} 
En este artículo destacamos la importancia de los conceptos en la enseñanza de la Mecánica, que es una de las partes de la Física. La Mecánica puede ser estudiada partiendo de las leyes de Newton o alternativamente usando los principios de conservación.

Generalmente los libros de texto no profundizan en la explicación detallada de los conceptos y más bien se limitan a presentar las leyes apoyándose en su formulación Matemática. La razón de lo anterior radica sustancialmente en que estos textos se elaboran para ser usados en cursos de Física Básica para estudiantes de Ingeniería.

\section{Los conceptos de espacio y tiempo en Mecánica}

El concepto de espacio en la Mecánica está relacionado con el contenido de las leyes del movimiento de Newton indicando que el mundo real es tridimensional y los cuerpos están contenidos allí como los objetos están dentro de un recipiente. Desde este punto de vista el espacio se generó antes que la materia siguiendo el paradigma de la creación Divina del Universo.

Adicionalmente el concepto Newtoniano de espacio anexa las características de inmóvil, ilimitado, homogéneo, inmutable y euclidiano implicando de esta forma su carácter absoluto y ligándolo a la existencia de un ser supremo. Newton también introduce el concepto de tiempo absoluto como algo verdadero y matemático, que fluye siempre de manera igual y sin relación a nada externo. Esta definición de tiempo absoluto no implica ni movimiento ni cambio en general. El concepto Newtoniano de espacio y tiempo no muestra ninguna conexión entre ellos, es decir, se consideran mutuamente independientes entre sí. Este esquema conceptual entra en conflicto con los desarrollos de la Teoría Especial de la Relatividad de Einstein.

\section{Los conceptos de materia y energía en Mecánica}

De la misma manera como los conceptos de espacio y tiempo son separables en Mecánica, sin guardar ninguna conexión, las nociones de materia y energía no tienen nada en común desde el punto de vista Newtoniano. El concepto de materia en la mecánica clásica se toma muy ligeramente sin entrar a profundizar acerca de sus implicaciones. Si tomamos cualquier texto de Física Elemental, encontramos indistintamente que, definen materia como «todo aquello de que están hechos los cuerpos».

La anterior definición de materia, introducida en los cursos de Mecánica, parece ser que penetra profundamente en las mentes de nuestros estudiantes y no existe forma de que ellos cambien la noción. A nuestro entender esta definición no es la más precisa y adecuada a este nivel, pues es muy macroscópica e inconexa.

El concepto Newtoniano de la energía está íntimamente relacionado con el trabajo efectuado por una fuerza. La energía se conceptualiza como: "la capacidad que tiene un sistema para realizar trabajo».

En la Teoría de la Relatividad Especial, los conceptos de materia y energía se complementan debido al principio de equivalencia entre masa y energía $\left(E=m c^{2}\right)$ siendo $c$ la velocidad de la luz. 
Marcos de referencia, sistemas de referencia e inercia

Un marco de referencia es un lugar en el espacio que se usa para medir las posiciones, las velocidades y aceleraciones de un sistema de partículas. El marco de referencia escogido por un observador (persona que efectúa mediciones) es arbitrario.

El marco de referencia se convierte en sistema de referencia cuando se escogen coordenadas adecuadas para describir el movimiento de un sistema físico. Las coordenadas escogidas dependen de la simetría del problema estudiado (simetría cartesiana, simetría esférica, simetría cilíndrica, etc.).

Para que las leyes del movimiento de Newton adquieran significado, es necesario tener un sistema de referencia con características acordes a los conceptos de espacio absoluto, tiempo absoluto e inercia. La búsqueda de un sistema de referencia absoluto, el éter lumínico, arrojó resultados negativos y esto condujo al nacimiento de la Teoría Especial de la Relatividad que desvirtúa la existencia de sistemas de referencia absolutos.

El concepto Newtoniano de tiempo absoluto, el mismo para todos los observadores, no resistió la prueba de los experimentos llevados a cabo por relojes idénticos colocados en naves que circundaban la tierra y en sitios fijos colocados en la superficie terrestre. Para los objetos más rápidos (comparados con la velocidad de propagación de la luz) y muy pequeños, los conceptos Newtonianos de espacio, tiempo, materia y energía no funcionan y es necesario redefinirlos.
La primera ley de Newton que determina el estado de movimiento o de reposo de una partícula, por la acción o carencia de una fuerza, incorpora la necesidad de tener marcos de referencia que se muevan entre sí a velocidad relativa constante para que las leyes de Newton tengan validez. En otras palabras, las leyes de Newton, sólo son válidas en estos marcos de referencia y ellos se denominan «marcos de referencia inerciales».

El concepto de partícula, que nació en la formulación Newtoniana de la Mecánica, se entiende como la de un punto geométrico en el espacio carente de estructura interna. Esta definición, en el marco de una formulación macroscópica de la materia, aunque útil debe ser revisada.

\section{Conclusiones}

Los conceptos de espacio, tiempo, materia, energía, partícula, inercia, marcos de referencia inerciales, deben ser analizados para mirar su verdadero alcance en otras ramas de la Física distintas a la Mecánica. Muchos conceptos tienen una aplicación parcial en la Teoría de la cual provienen y pueden ser llevados a un nuevo modelo pero efectuando a cabo una redefinición.

Para comprender las leyes y principios de la Física, es necesario entender los conceptos y las definiciones, las cuales en la mayoría de las ocasiones no guardan ninguna relación con la formulación matemática de la Teoría. Es erróneo pensar que un conjunto de leyes expresadas por ecuaciones, puedan ser entendidas y aplicadas acertadamente mediante un simple razonamiento matemático. 


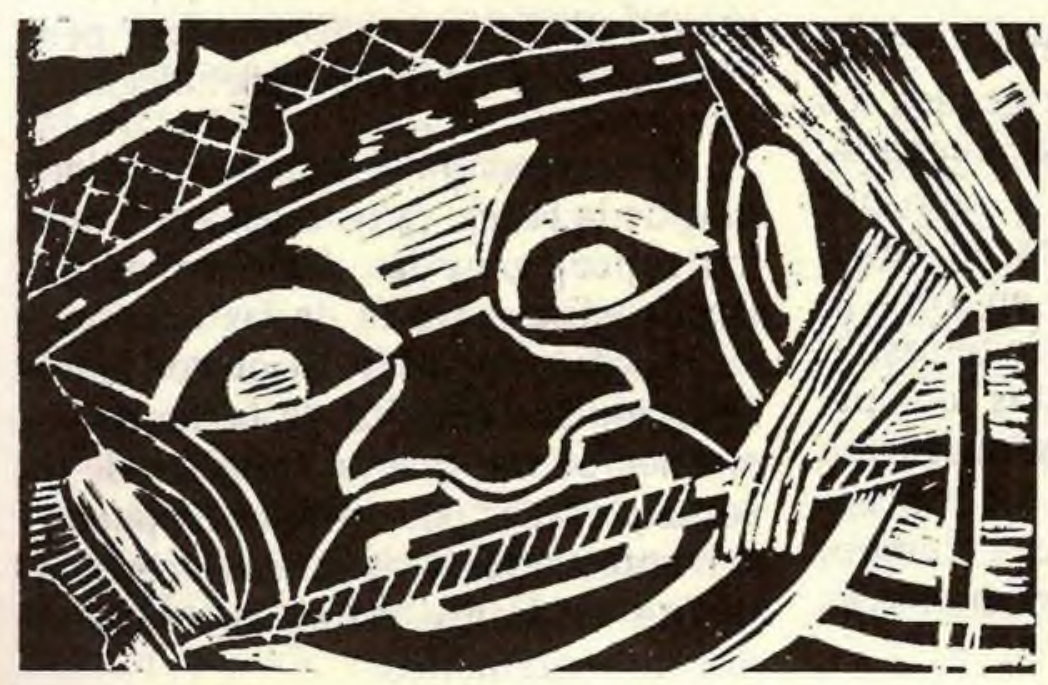

Ilustración: Rosalba Cortés Arteaga 